\title{
BMJ Open Parental and healthcare professional concern in the diagnosis of paediatric sepsis: a protocol for a prospective multicentre observational study
}

\author{
Zoe Sever (D) , 'Luregn J Schlapbach (D) , ${ }^{2,3}$ Melanie Jessup (D) , \\ Shane George (D) , ${ }^{4,5}$ Amanda Harley (1) 1,2,4,6
}

To cite: Sever Z, Schlapbach LJ, Jessup M, et al. Parental and healthcare professional concern in the diagnosis of paediatric sepsis: a protocol for a prospective multicentre observational study. BMJ Open 2021;11:e045910. doi:10.1136/ bmjopen-2020-045910

- Prepublication history for this paper is available online. To view these files, please visit the journal online (http://dx.doi org/10.1136/bmjopen-2020045910).

Received 26 October 2020 Accepted 25 August 2021

\section{Check for updates}

(c) Author(s) (or their employer(s)) 2021. Re-use permitted under CC BY-NC. No commercial re-use. See rights and permissions. Published by BMJ.

For numbered affiliations see end of article.

Correspondence to Amanda Harley; Amanda.Harley@health.qld. gov.au

\section{ABSTRACT}

Introduction Paediatric sepsis is a major contributor to morbidity and mortality worldwide. Assessing concern from parents and healthcare professionals to determine disease severity in a child evaluated for sepsis remains a field requiring further investigation. This study aims to determine the diagnostic accuracy of parental and healthcare professional concern in the diagnosis of children evaluated for sepsis.

Methods and analysis This prospective multicentre observational study will be conducted over a 24-month period in the paediatric emergency department (ED) at two tertiary Australian hospitals. A cross-sectional survey design will be used to assess the level of concern in parents, nurses and doctors for children presenting to $E D$ and undergoing assessment for sepsis. The primary outcome is a diagnosis of sepsis, defined as suspected infection plus organ dysfunction at time of survey completion. Secondary outcomes include suspected or proven infection and development of organ dysfunction, defined as a Paediatric Sequential Organ Failure Assessment Score $>0$, within 48 hours of presentation, paediatric intensive care unit admission, confirmed or probable bacterial infection independent of organ dysfunction, and hospital length of stay.

Ethics and dissemination Ethics approval was obtained from Children's Health Queensland's Human Research Ethics Committee (HREC/17/QRCH/85). Findings will be shared with relevant stakeholders and disseminated via conferences and peer-reviewed journals

Trial registration number $\mathrm{WHO}$ Universal Trial Number, U1111-1256-4537; ANZCTR number, ACTRN1262000134092.

\section{INTRODUCTION}

Sepsis is a major contributor to morbidity and mortality in children worldwide. ${ }^{1}$ The WHO recently identified sepsis as a key health priority, outlining the high global burden of this time critical and often preventable disease. ${ }^{2}$ While the latest definition of paediatric sepsis dates back to $2005,{ }^{3}$ the definition of sepsis in adults was redefined in 2016 as 'life threatening organ dysfunction caused by
Strengths and limitations of this study

- This is expected to be the largest study to date, which assesses the diagnostic accuracy of parental and healthcare professional concern in recognising sepsis within the Emergency Department.

- Prospective assessment of parental, nursing and medical concern will be undertaken both quantitatively and qualitatively.

- The study will be emedded in an institutional paediatric sepsis pathway, reducing barriers for staff engagement.

- Consideration for other sources of diagnostic bias as a result of referral, previous history and concomitant interventions is required.

a dysregulated host response to infection'. ${ }^{4}$ Globally, close to 50 million patients suffer from sepsis each year, with over 10 million sepsis-related deaths, ${ }^{5}$ the highest incidence affecting infants and children. While the highest burden related to sepsis affects lowincome and middle-income settings, sepsis remains among the leading causes of (potentially preventable) morbidity and mortality in high-income countries too, accounting for a total cost of US $\$ 7.31$ billion in the USA alone. ${ }^{6}$ These high economic costs, along with the persistently high prevalence and morbidity of paediatric sepsis, highlight the urgent need for further research into earlier sepsis recognition.

Prompt identification is well recognised as fundamental for early intervention and treatment of sepsis. In a large retrospective study of children with sepsis, the delay in the administration of a sepsis treatment bundle consisting of intravenous antibiotics, fluids and blood cultures was associated with a significant increase in mortality. ${ }^{7}$ The majority of paediatric sepsis deaths occur within the first 48 
hours of initial admission to the intensive care unit, ${ }^{8}$ emphasising the need for prompt recognition and resuscitation. The new Surviving Sepsis Guidelines ${ }^{9}$ further emphasise the need for early detection as it is a critical survival factor for paediatric sepsis, with timely and appropriate initiation of interventions being linked to improved patient outcomes.

Paediatric sepsis often starts as an insidious condition, which poses many challenges for healthcare professionals to accurately and timely diagnose it. This is due to the vague and non-specific nature of the disease, coupled with a relatively low incidence rate compared with the number of children presenting to the emergency department (ED) with febrile illness. ${ }^{10}$ Consequently, the risk for a missed diagnosis is high and subsequent repercussions are potentially lifelong or fatal. ${ }^{11}$ In its early stages, sepsis often resembles many other common febrile illnesses with the clinical signs of fever, tachycardia and tachypnoea. ${ }^{12}$ Parents, as experts of their child, may be valuable in the identification of sepsis and discrimination of the condition from other milder illnesses.

Observational studies suggest that parents may recognise illness severity before nurses and doctors, independent of key clinical signs. ${ }^{13}$ Root cause analyses and anecdotal data after fatal paediatric sepsis outcomes established that children often re-presented several times and parents commonly indicated concerns that the 'illness was different'. ${ }^{13}$ A more holistic and family-centred-care approach incorporating collaboration between the child's family and treating team has the potential to enhance the timely recognition of sepsis. ${ }^{14}$

The current diagnostic approach for sepsis rely predominately on clinician-guided assessment or physiologybased tools, which pose numerous challenges due to the complex nature of paediatric physiology. ${ }^{15}$ While the search for more precise biomarkers for sepsis continues, little is known in relation to using concern as a diagnostic tool to aid in earlier recognition. ${ }^{16}$ In addition to parental concern, the gut feeling or intuition of healthcare professionals may contribute to the recognition of sepsis. ${ }^{17}$ In the primary care setting, a gut feeling that 'something was wrong' reported by clinicians was linked with a high specificity and positive likelihood ratio for serious bacterial infections. ${ }^{18}$ The inclusion of parental and healthcare professional concern in the diagnostic approach has the potential ability to improve specificity, thereby increasing sepsis recognition.

We hypothesise that the inclusion of parental and healthcare professional concern in the ED will improve diagnostic accuracy and early recognition of paediatric sepsis. The main objective of this study is to determine the diagnostic accuracy of concern levels in parents, doctors and nurses to recognise paediatric sepsis in a prospective multicentre observational study.

\section{METHODS AND ANALYSIS}

\section{Study design}

This prospective multicentre observational cohort study will use a cross-sectional survey tool designed to independently assess the level of concern in parents, nurses and doctors for children who present to the ED and are evaluated for sepsis. The planned duration for the project will be at least 24 months for recruitment with 6 months for data cleaning, analyses and write up. The study started recruitment in December 2018 . This study has been designed to fulfil criteria for Standards for Reporting Diagnostic accuracy studies (STARD).$^{19}$

\section{Study setting}

This study will be conducted across the dedicated paediatric EDs at two tertiary Australian hospitals: Queensland Children's Hospital, which receives approximately 6600 presentations each month, and Gold Coast University Hospital, which receives approximately 2300 paediatric presentations each month.

\section{Participants}

Eligible participants will be children aged between 30 days and 18 years presenting to the ED and evaluated for sepsis via the institutional sepsis pathway and/or undergoing blood culture sampling for suspected infection (table 1).

\section{Study criteria}

\section{Test methods}

The study surveys have been designed for parents, nurses and doctors, incorporating both quantitative and qualitative measures (figures 1-3). To ensure consistent comparison, all surveys have the same basic design and content, with minor adaptions on the parent/carer survey to reflect the participant role (parent vs nurse/doctor). Participants are asked to rate the degree to which they agree or disagree with a statement or question using a

\begin{tabular}{ll|}
\hline Table 1 Study inclusion and exclusion criteria \\
\hline Inclusion criteria & Exclusion criteria \\
\hline Child aged 30 days-18 & Parents who do not speak \\
years old & English \\
Presented to ED & Children with high \\
Evaluated for sepsis on & suspicion of SARS-CoV-2 \\
the sepsis pathway and/ & infection* \\
or having blood culture & Patients in clinical areas \\
sampling & outside the ED such as the \\
Survey completed during & paediatric intensive care \\
ED stay, aiming to be & unit \\
completed at time closest & \\
to triage presentation & \\
Parent/caregiver attending & \\
with child, treating doctor & \\
and/or nurse available for & \\
survey & \\
\hline
\end{tabular}

${ }^{*}$ Research governance did not permit researchers to risk exposure to SARS-CoV-2 infection. 


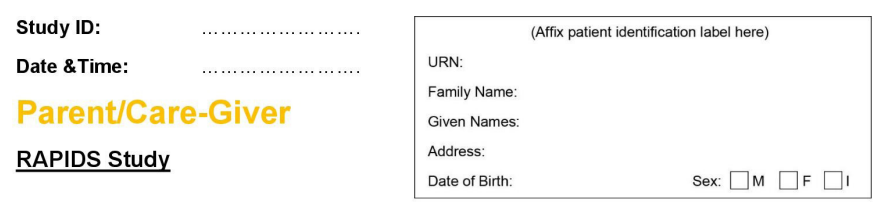

We would like to ask you to participate in this study. We are interested in measuring the parental concern as a tool to predict how severe a child's illness may be No one knows your child better than you so we ask that you please complete this short survey to indicate if/what concerns you most about your child's illness. By completing this survey you are consenting to us analysing this data together with information from the medical health record for research purposes.

1. Please place an ' $\mathrm{X}$ ' in the below fields to indicate your current level of concern regarding the severity of your child's illness:

$\begin{aligned} & \text { Not } \\ & \text { Concerned }\end{aligned}$
2. Do you have a gut feeling that something is wrong with your child? Please place an ' $\mathrm{X}$ ' in the below fields
to indicate your current level of concern regarding the severity of your child's illness:
I feel things
are ok
3. Please place an ' $\mathrm{X}$ ' in the below fields to indicate how different is your child's behaviour right now compared
to normal?
Normal
Behaviour for
Child

4. What are the symptoms/behaviours that your child displayed, which prompted you to bring your child to hospital? Please list as many as possible:

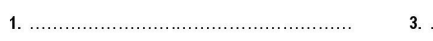

2.

5. What was the symptom that concerned you the most?

6. Please place an ' $\mathrm{X}$ ' in the below fields to indicate how severe you feel your child's illness is today?
Mildly
Unwell
7. Please place an ' $\mathrm{X}$ ' in the below fields to indicate how unwell you feel your child is today compared to how
unwell they have been in the past:
$\begin{aligned} & \text { Mild IIIness for } \\ & \text { Child }\end{aligned}$

Figure 1 Parental survey.

5-point Likert scale, ${ }^{20}$ followed by two free text questions. The surveys were piloted 20 times to ensure questionnaire feasibility. Job title and years of experience for participating doctors and nurses will be collected. ${ }^{17}$

The surveys will be distributed to one of the child's parents/caregivers, nurse and doctor on presentation and will be completed during the ED stay. The distribution of these surveys will occur 7 days a week 24 hours a day through the ED staff with support from the dedicated research team. We aim for surveys to be completed at time closest to triage and within 4 hours from initial presentation. This window for survey administration was determined based on the current Australian National Emergency Assess Target guidelines, which stipulate that patients must be admitted, discharged or transferred from ED within 4 hours of initial presentation. ${ }^{21}$ These surveys will be embedded within the Queensland paediatric sepsis pathway, which was developed and implemented across Queensland paediatric EDs from 2018.

\section{Sample size}

A minimum of 450 patients will be recruited over the two sites. This minimum sample size was selected based on a sample size calculation, which revealed that with an expected

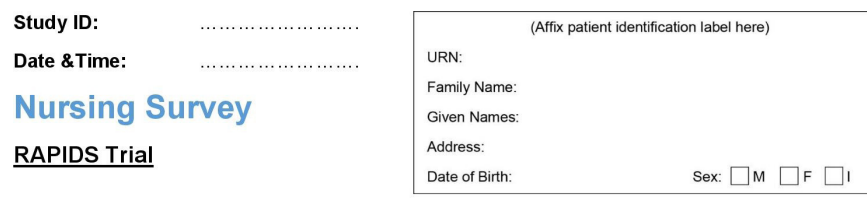

We would like to ask you to participate in this study. We are interested in measuring the treating nurse's level of concern as a tool to predict how severe a child's illness may be. We ask that you please complete this short survey to indicate what concerns you most about your patient's illness. By completing this survey you are consenting to us including this data for research purposes.

Job Title (RN/CN):

Years of Experience:

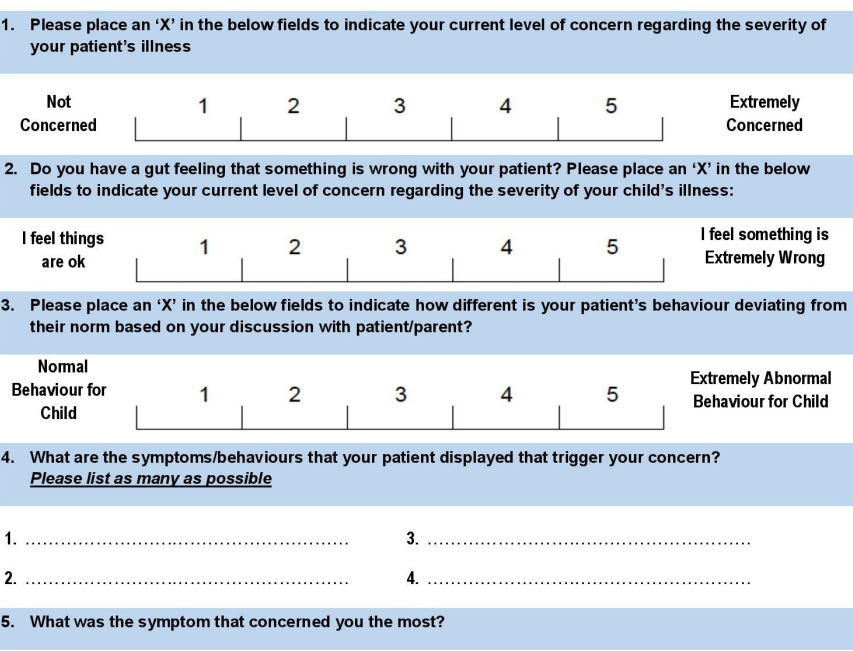

6. Please place an ' $\mathrm{X}$ ' in the below fields to indicate how severe you feel your patient's illness is: \begin{tabular}{l|l|l|l|l|l|l|l|l} 
Mildly \\
Unwell
\end{tabular}

Figure 2 Nursing survey.

prevalence of $10 \%$ and an expected improvement in sensitivity from 0.6 to 0.8 , a sample size of 450 is needed. ${ }^{22}$

\section{Data collection}

The patient demographics, information regarding the presentation and illness severity at baseline will be collected from the medical record. In addition, the worst measure of physiological parameters and maximum level of support during the first 48 hours will be captured. Illness severity will be determined using the Paediatric Sequential Organ Failure Assessment (pSOFA) Score. ${ }^{23}$ Data will be recorded into a secure RedCap case report form.

\section{Analysis plan}

Suspected or proven infection in the presence of organ dysfunction, defined as a pSOFA Score $>0$ at time of assessment, is defined as the primary outcome. Given the ongoing controversy around paediatric sepsis definitions,${ }^{12}$ sensitivity analyses with organ dysfunction defined as per the 2005 International Paediatric Definitions Consensus Conference will be performed. ${ }^{324}$ Secondary outcomes are defined as: (a) suspected or proven infection and development of organ dysfunction, defined as a pSOFA Score $>0$, within 48 hours of presentation; (b) admission to the Paediatric Intensive Care Unit; (c) confirmed or probable bacterial infection independent 


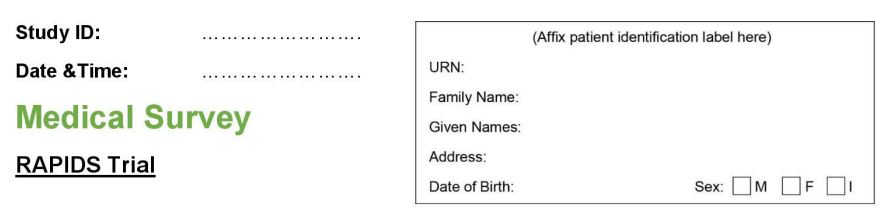

We would like to ask you to participate in this study. We are interested in measuring the treating doctor's level of concern as a tool to predict how severe a child's illness may be. We ask that you please complete this short survey to indicate what concerns you most about your patient's illness. By completing this survey you are consenting to us including this data for research purposes.
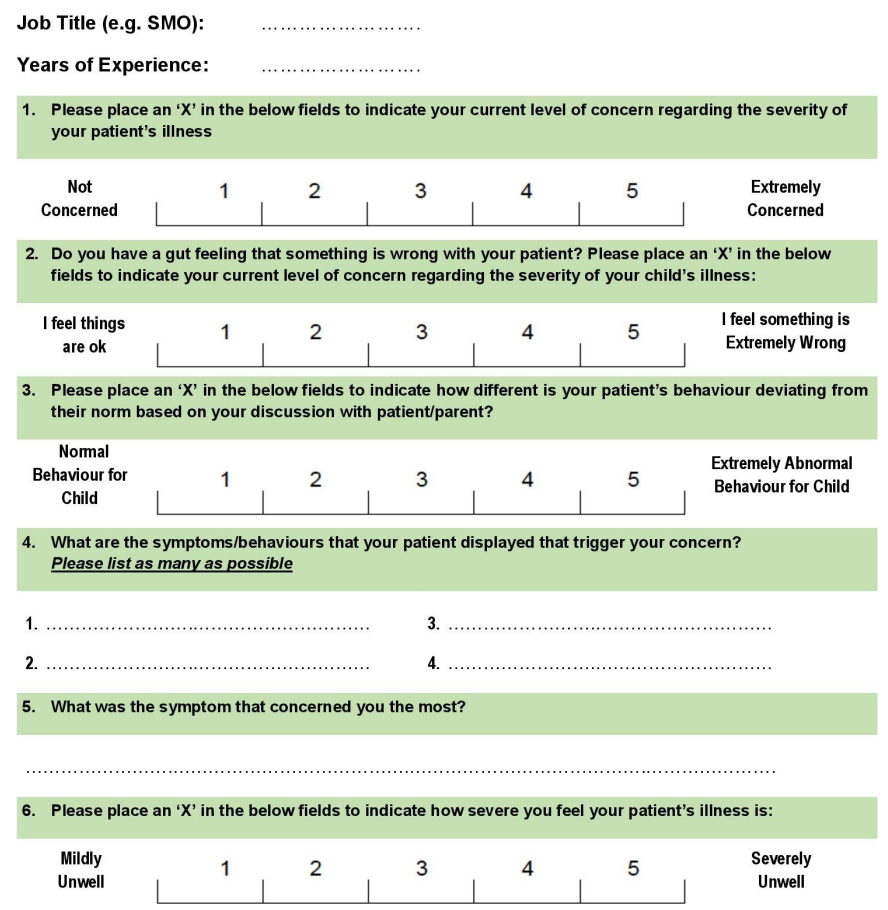

Figure 3 Medical survey.

of organ dysfunction and (d) hospital length of stay. The likelihood of bacterial versus viral infection will be determined by an independent assessor using all available laboratory, microbiological and clinical information with adjudication of patients. ${ }^{25}$ Bacterial infection will be categorised as confirmed bacterial infection (positive microbiological cultures compatible with the clinical syndrome, and decision by the treating physician to treat for at least 5 days or until death with antibiotics) or probable bacterial infection (negative microbiological cultures in the presence of a clinical syndrome of bacterial infection and increased $\mathrm{C}$ reactive protein, and decision by the treating physician to treat for at least 5 days or until death with antibiotics). Viral infection will be categorised as probable viral infection (negative microbiological tests in the presence of a clinical syndrome of viral infection such as bronchiolitis) or proven viral infection (positive microbiological testing in the presence of a clinical syndrome of viral infection). If the presentation is determined to be of non-infectious or unknown origin, it will be classed as infection of uncertain origin, or as non-infectious conditions.

Descriptive analyses will report on the demographics and baseline patient features. Description on the level of completeness of the surveys (parental, nursing and medical) will be provided and any differences in demographics will be investigated between children who have completed surveys from all three participant groups and those who have missing surveys.

To assess the relationship between the concern ratings and outcome, first, an exploratory factor analysis will be performed on the four concern questions assessed in the surveys to determine whether the questions are measuring the same latent construct ('concern') or if more than one construct is present. In addition, the internal consistency and inter-rater reliability of the concern questions will be assessed using Cronbach's alpha and the intraclass correlation using a one-way random effects model, respectively. Based on the results of the factor analysis, a factor score will be created and used as a measure of concern in the regression models. In addition, the relationship between the four individual concern questions with the primary outcome will be assessed through bivariate logistic regression models. The question that provides the best prediction of sepsis will be identified as the one that has the highest unadjusted OR and area under the receiver operating characteristic curve (AUROC), with 95\% CIs being reported alongside all effects.

Next, regression models will be derived for the primary and secondary outcomes to assess the associations between the concern factor score and the 'best' concern question with the outcome. Other demographic characteristics and physiological variables, which are associated with the outcomes of interest, will be included in the model as control variables. The AUROC, sensitivity, specificity, negative and positive likelihood ratios (along with associated 95\% CIs) will be calculated to assess model fit and predictive performance.

All regression modelling will be performed on each of the three responder cohorts separately (ie, children with a parental survey, children with a nurse survey and children with a medical survey completed) to identify whether the effect of concern on the outcomes is dependent on the responder. $\mathrm{P}$ values below 0.05 will be considered as statistically significant. All analyses will be performed by an expert statistician using R. ${ }^{26}$

A preplanned secondary analysis will assess qualitative data from the survey free text questions using the framework method. ${ }^{27}$ These free texts will then be examined and sorted into multiple categories to determine commonalities and differences. These categories will then be sorted into themes for the three groups: parents, doctors and nurses. ${ }^{27}$

\section{Strengths}

A strength of this study is its prospective observational study design with a large multicentre cohort of children evaluated for sepsis. In contrast to previous studies, which more broadly captured serious bacterial infections ${ }^{18}$ or fever, ${ }^{28}$ the present study captures sepsis defined as suspected/proven infection with organ dysfunction as the main outcome. The study design enables assessment of the role of parental and healthcare professional concern in diagnosing paediatric sepsis and compares 
the respective diagnostic accuracies with the diagnostic performance of the routine diagnostic process.

This study aims to address an established gap regarding the significance of parental and healthcare professional concern in predicting disease severity in children with infection. Outcomes can inform the design of improved sepsis recognition tools. While the study will be conducted within the ED, findings relating to the use of concern as a red flag and a prompt for further investigation and assessment could be translated into other clinical settings.

\section{Limitations}

This study presents several limitations. It is expected that some patients will only have partial sets of surveys completed due to circumstances such as a parent being deemed unfit to complete a research survey for various reasons, or the attending nurse or doctor not completing a survey. The incomplete sets of surveys for patients are anticipated given the pragmatic nature of the study and will be a consideration when conducting analysis and reporting. Bias could occur through children who are more clinically well having a greater number of concern surveys completed, as opposed to more clinically unwell children, whereby parents may be too distressed and healthcare professionals otherwise occupied treating the child. ${ }^{29}$ Standardised dissemination of study education will aim to reduce potential bias related to variances on how the surveys are administered to parents, doctors and nurses. Implementation of an educational script will eliminate the use of words such as sepsis, organ dysfunction or death, which may potentially heighten concern levels or result in changes to concern. Finally, the study will be performed in two sites working within the same healthcare system in a high-income country, and, hence, similar studies in low-income and middle-income settings will be required to assess generalisability.

\section{ETHICS AND DISSEMINATION}

Informed verbal consent will be gained from the parent/ caregiver, nurse and doctor at the time of survey administration. It will be reiterated to all parties that they have the right to refuse participation at initial time of consent or withdraw at any stage without affecting patient care or their employment, as applicable. The survey and study design have been approved by the Children's Health Queensland's Human Research Ethics Committee (HREC/17/QRCH/85).

Findings will be shared with relevant stakeholders and disseminated via conferences and peer-reviewed journals.

\footnotetext{
Author affiliations

${ }^{1}$ School of Nursing, Midwifery and Social Work, The University of Queensland, Brisbane, Queensland, Australia

${ }^{2}$ Child Health Research Centre and Paediatric Intensive Care Unit, The University of Queensland and Queensland Children's Hospital, Brisbane, Queensland, Australia

${ }^{3}$ Department of Intensive Care and Neonatology, Children's Research Center, University Children's Hospital Zürich, Zürich, Switzerland
}

${ }^{4}$ Departments of Emergency Medicine and Children's Critical Care Service, Gold Coast University Hospital, Southport, Queensland, Australia

${ }^{5}$ Faculty of Health, School of Medicine and Menzies Health Institute Queensland, Griffith University, Gold Coast, Queensland, Australia

${ }^{6}$ Critical Care Nursing Management Team, Queensland Children's Hospital, Brisbane, Queensland, Australia

Twitter Zoe Sever @SeverZoe, Shane George @shanegeorge77 and Amanda Harley @theamandaharley

Contributors LJS conceived the project and provided the technical and intellectual inputs supporting ZS and AH in writing this manuscript and approved it for submission. ZS produced the first draft and subsequent drafts of the paper, supported by AH. AH and ZS lead central coordination and contributed to the design of the analysis plan. ZS, LJS, MJ, SG and AH were involved in the review and provided inputs on the manuscript. All authors reviewed and approved various drafts and the final paper.

Funding This work is supported by the Emergency Medicine Foundation, Queensland, Australia EMJS-327R31-2019-HARLEY. LJS was supported by a Practitioner Fellowship from NHMRC and by the Children's Hospital Foundation, Brisbane, Australia.

Competing interests None declared.

Patient consent for publication Not required.

Provenance and peer review Not commissioned; externally peer reviewed.

Open access This is an open access article distributed in accordance with the Creative Commons Attribution Non Commercial (CC BY-NC 4.0) license, which permits others to distribute, remix, adapt, build upon this work non-commercially, and license their derivative works on different terms, provided the original work is properly cited, appropriate credit is given, any changes made indicated, and the use is non-commercial. See: http://creativecommons.org/licenses/by-nc/4.0/.

\section{ORCID iDs}

Zoe Sever http://orcid.org/0000-0003-1508-5716

Luregn J Schlapbach http://orcid.org/0000-0003-2281-2598

Melanie Jessup http://orcid.org/0000-0002-5243-9117

Shane George http://orcid.org/0000-0002-8880-1245

Amanda Harley http://orcid.org/0000-0003-2665-9324

\section{REFERENCES}

1 Fleischmann-Struzek C, Goldfarb DM, Schlattmann P, et al. The global burden of paediatric and neonatal sepsis: a systematic review. Lancet Respir Med 2018;6:223-30.

2 Kissoon N, Reinhart K, Daniels R, et al. Sepsis in children: global implications of the world health assembly resolution on sepsis. Pediatr Crit Care Med 2017;18:e625-7.

3 Goldstein B, Giroir B, Randolph A, et al. International pediatric sepsis consensus conference: definitions for sepsis and organ dysfunction in pediatrics. Pediatr Crit Care Med 2005;6:2-8.

4 Singer M, Deutschman CS, Seymour CW, et al. The third International consensus definitions for sepsis and septic shock (Sepsis-3). JAMA 2016;315:801-10.

5 Rudd KE, Johnson SC, Agesa KM, et al. Global, regional, and national sepsis incidence and mortality, 1990-2017: analysis for the global burden of disease study. Lancet 2020;395:200-11.

6 Carlton E. Cost of Pediatric Severe Sepsis Hospitalizations | Critical Care Medicine | JAMA Pediatrics | JAMA Network [Internet], 2019. Available: https://jamanetwork.com/journals/jamapediatrics/articleabstract/2748380 [Accessed 07 Apr 2020].

7 Evans IVR, Phillips GS, Alpern ER, et al. Association between the new York sepsis care mandate and in-hospital mortality for pediatric sepsis. JAMA 2018;320:358-67.

8 Schlapbach LJ, MacLaren G, Festa M, et al. Prediction of pediatric sepsis mortality within $1 \mathrm{~h}$ of intensive care admission. Intensive Care Med 2017;43:1085-96.

9 Weiss SL, Peters MJ, Alhazzani W, et al. Surviving sepsis campaign international guidelines for the management of septic shock and sepsis-associated organ dysfunction in children. Pediatr Crit Care Med 2020;21:e52-106.

10 Schlapbach LJ, Weiss SL, Wolf $J$. Reducing collateral damage from mandates for time to antibiotics in pediatric Sepsis-Primum non nocere. JAMA Pediatr 2019;173:409-10. 
11 Schlapbach LJ, Straney L, Alexander J, et al. Mortality related to invasive infections, sepsis, and septic shock in critically ill children in Australia and New Zealand, 2002-13: a multicentre retrospective cohort study. Lancet Infect Dis 2015;15:46-54.

12 Schlapbach LJ, Kissoon N. Defining pediatric sepsis. JAMA Pediatr 2018;172:313-4.

13 Launay E, Gras-Le Guen C, Martinot A, et al. Why children with severe bacterial infection die: a population-based study of determinants and consequences of suboptimal care with a special emphasis on methodological issues. PLoS One 2014;9:e107286.

14 Dennis C, Baxter P, Ploeg J, et al. Models of partnership within family-centred care in the acute paediatric setting: a discussion paper. J Adv Nurs 2017;73:361-74.

15 Roland D, Snelson E. 'So why didn't you think this baby was ill?' Decision-making in acute paediatrics. Arch Dis Child Educ Pract Ed 2019;104:43-8.

16 Thompson M, Van den Bruel A, Verbakel J, et al. Systematic review and validation of prediction rules for identifying children with serious infections in emergency departments and urgent-access primary care. Health Technol Assess 2012;16:1-100.

17 Roland D. Have we forgotten to teach how to think? Emerg Med J 2017;34:68-9.

18 Van den Bruel A, Thompson M, Buntinx F, et al. Clinicians' gut feeling about serious infections in children: observational study. BMJ 2012;345:e6144.

19 Cohen JF, Korevaar DA, Altman DG, et al. STARD 2015 guidelines for reporting diagnostic accuracy studies: explanation and elaboration. BMJ Open 2016;6:e012799.
20 Sullivan GM, Artino AR. Analyzing and interpreting data from likerttype scales. J Grad Med Educ 2013;5:541-2.

21 Sullivan C, Staib A, Khanna S, et al. The National emergency access target (NEAT) and the 4-hour rule: time to review the target. Med $J$ Aust 2016;204:354.

22 Bujang MA, Adnan TH. Requirements for minimum sample size for sensitivity and specificity analysis. J Clin Diagn Res 2016;10:YE01-6.

23 Matics TJ, Sanchez-Pinto LN. Adaptation and validation of a pediatric sequential organ failure assessment score and evaluation of the Sepsis-3 definitions in critically ill children. JAMA Pediatr 2017;171:e172352

24 Gebara BM. Values for systolic blood pressure. Pediatr Crit Care Med 2005;6:500.

25 Herberg JA, Kaforou M, Wright VJ, et al. Diagnostic test accuracy of a 2-Transcript host RNA signature for discriminating bacterial vs viral infection in febrile children. JAMA 2016;316:835-45.

$26 \mathrm{R}$ Core Team. A language and environment for statistical computing. R Foundation for Statistical Computing [Internet], 2019. Available: https://www.R-project.org/

27 Gale NK, Heath G, Cameron E, et al. Using the framework method for the analysis of qualitative data in multi-disciplinary health research. BMC Med Res Methodol 2013;13:117.

28 Urbane UN, Gaidule-Logina D, Gardovska D, et al. Value of parental concern and clinician's gut feeling in recognition of serious bacterial infections: a prospective observational study. BMC Pediatr 2019;19:219.

29 Hammer GP, du Prel J-B, Blettner M. Avoiding bias in observational studies. Dtsch Arztebl Int 2009;106:664-8. 